XXV.

\title{
II. Die Vorhofswasserleitung und ihre Rolle bei Labyrintheiterungen.
}

Von

Dr. Max Goerke in Breslau.

In dem Kapitel der otogenen Hirnkomplikationen war als eine der ersten und bedeutungsvollsten Fragen, die nach der Art und Weise des Übergangs einer Eiterung rom Mittelohre nach dem Schädelinnern zu erledigen: die Wichtigkeit dieser Frage, deren Beantwortung dem Operateur nicht minder am Herzen lag, als dem pathologischen Anatomen, wird durch eine Fülle von Arbeiten illustriert, die sich an ihre Lösung auf verschiedenem, mehr oder minder aussichtsvollem Wege heranwagten. Nachdem nun einmal - und zwar gleichermaßen vom Kliniker wie vom Pathologen - das Labyrinth als eine der wichtigsten Etappen auf dieser Übergangsbahn vom Mittelobr nach dem Gehirn erkannt war, galt es in zweiter Reihe - und diese Aufgabe fiel vornehmlich dem Anatomen zu - klarzustellen, welche präformierten oder nicht präformierten Bahnen der Infektion den Einbruch aus dem Labyrinthe nach innen gestatteten. Nachdem nun des weiteren sehr bald die Erkenntnis gewonnen war, dak hier fast ausschließlich die anatomisch präformierten Wege in Betracht kommen, lag der Gedanke nahe, durch kritische Prïfung und Zusammenstellung des in der Literatur reichlich niedergelegten Materials die Unterfrage zu erledigen, wie sich die einzelnen dieser der Infektion stets offen liegenden Bahnen hinsichtlich der Häufigkeit ihrer Beteiligung d. h. klinisch gesprochen, linsichtlich ihrer Gefährlichkeit zu einander verhalten. 
Da kommt nun die Zusammenstellung von Boesch ${ }^{1}$ ) zu dem überraschenden Ergebnis, daß in 33,84\% aller Fälle überhaupt (in $40 \%$ :, wenn die präformierten Bahnen allein berticksichtigt werden) der aquaeductus vestibuli der schuldige Teil ist, während anf den aquaeductus cochleae nur $3 \%$ fallen. Ich bezeichne das Ergebnis als ein überraschendes: denn gerade das umgekehrte Verhältnis müßten wir voraussetzen, in Berücksichtigung der anatomischen Tatsache, daß der aquaeductus vestibuli blind endigt, durch ein festes Durablatt gegen die Arachnoidealräume abgeschlossen ist, während die Schneckenwasserleitung eine stets offene direckte Verbindung zwischen jenen und dem Labyrinthe darstellt. So habe ich bei meinen Untersuchungen über die Genickstarre-Labyrinthitis in mehreren Fällen mit Sicherheit den aquaeductus cochleae als Passage für den Eiter, der hier allerdings die umgekehrte Richtung einschlägt, feststellen können, während nur in einem einzigen Falle die "Möglichkeit" eines Durchbruchs durch die Vorhofswasserleitung vorhanden war. Boesch selbst erscheint das Ergebnis seiner prozentualen Berechnung so befremdlich, daß er nach einer plausiblen Erklärung sucht: Er glaubt, daß der Infektionsweg durch den aquaeductus cochleae "wegen der geringen örtlichen Zerstörungen am Übergangspunkte am schwersten nachzuweisen ist". Ich kann diese Behauptung nicht als zutreffend ansehen. Gerade der Übergang an dieser Stelle ist kaum zu übersehen, sofern man nur bei der mikroskopischen Untersuchung seine Aufmerksamkeit darauf richtet; makroskopische Veränderungen wird man freilich an jener Stelle häufig vermissen. Man kann meines Erachtens ohne weiteres annehmen, daß in vielen Fällen eine ungenügende mikroskopisebe Untersuchung die Duchbruchsstelle falsch lokalisiert hat; und man wird ferner vermuten dürfen, daß manche Autoren in ibren Fällen - hierher sind alle nur klinisch untersuchten zu rechnen - durch grob anatomische Veränderungen in der Gegend des Saccus endolymphaticus sich verführen ließen, hier die Durchbruchsstelle anzunehmen, die ganz wo anders su suchen war.

Diese Auffassung hat neuerdings auch $\mathrm{W}$ agener ${ }^{2}$ ) vertreten. Dieser hat den 21 Fällen von Boesch noch 10 weitere aus der

1) Bösch: Der Aquaednctus vestibuli als Infektionsweg. (Zeitschr. f. Otol. Bd. 50. S. 337.)

2) Wagener: Kritische Bemerkungen über das Empyem des Saccus endolymphaticus ete. (Arch. f. Ohreaheilk. Bd.68, S.273.) 
Literatur hinzugefügt und die Angaben in jedem einzelnen Falle auf ihre Beweiskraft hin kritisch geprüft. Er kommt zu dem Resultate, daß in keinem einzigen Falle mit Sicherheit der Übergang der Eiterung durch den aquaeductus vestibuli nachgewiesen ist; ja er geht noch weiter und behauptet: Mit Ausnahme eines einzigen Falles (des von Politzer) ') existiert kein zweiter, bei dem „einwandsfrei das Fortschreiten einer Eiterung vom Labyrinth zwischen die Durablätter" d. h. also das Zustandekommen eines sogenannten Empyems des Saceus endolymphaticus beobachtet ist. Ich kann an dieser Stelle auf eine Nachprüfung der Fälle bzw. auf eine Würdigung der Wagenerschen Kritik nicht eingehen; soviel ist sicher zuzugeben, daß den blob klinisch oder makroskopisch-anatomisch untersuchten Fällen keinerlei Beweiskraif zukommt. Wir werden sehen, wie schwierig die Beurteilung selbst bei genauer mikroskopischer Untersuchung sein kann.

An der Hand eines Falles von sicher festgestelltem Empyem des Saccus und sicherem Durchbruche des Eiters an dieser Stelle in die Meningen, des ersten als völlig einwandsfrei anzusehenden, wollen wir versuchen uns klarzumachen, worauf die Untersuchung in solchen und ähnlichen Fallen zu achten hat, und welche Schwierigkeiten sich einer richtigen Beurteilung entgegenstellen.

Krankengesch ichto (Auszug): Mann von 21 Jahren mit Ohrenlaufen links angeblich seit der Kindheit; Mai 1905 erhielt Patient einen Schlag gegen die rechte Warzenfortsatzgegend: darauf Eiteransammlung auf dem Warzenfortsatze und Operation im Krankenhause zu Lodz (Polen). Seitdem hat sich die Wuade hinter dem Ohre nicht ganz geschlossen. Beiderseits übelriechender Ausflub, $a b$ und zu leichte Kopfschmerzen, unbestimmtes Schwindelgefühl. Sonst hat Patient keine Krankheiten durchgemacht.

Status (27. Aug. 05): Rechts $1 \mathrm{~cm}$ hinter der Insertionslinie, parallel zu derselben verlaufend, eine $7 \mathrm{~cm}$ lange sehwielige, mit dem Knochen verwachsene Narbe. In der Mitte der Narbe, in der Höhe des Meatus externus, eine granulierende Stelle, an der 2 kleine Sequester heraushängen (Extraktion derselben). Die Sonde dringt in die Fistel obne Widerstand ein und gelangt in ca. $1 \mathrm{~cm}$ Tiefe in der Richtung nach oben anf rauhen Knochen. In der Tiefe des Gehörgangs. mäßige Mengen fötiden Sekrets; Gehörgang in der Tiefe durch allseitige hyperostotische Verdickung der Wandungen, besonders der vorderen und oberen, vollkommen stenosiert. Aus der Tiefe kommt sangumolentes fötides Sokret hervor. - Links ebenfalls schitziörmige Verengerung des Gehörgangs durch Hyperostose; aus dem Schlitze kommt fötides Sekret pulsierend hervor. Funktion: Rechts scharfe Flüstersprache ad coneham, links Flüsterzahlen in $10 \mathrm{~cm}$; Rinne beiderseits negativ; Knochenleitung verlängert; Weber unbestinmt.

Kein Exanthem, keine Drüsen; Augenbewegungen überallhin ausgiebig; Facialis beiderseits intakt: Patellarreflexe mäbig gesteigert; Sensibilität normal. Innere Organe ohne besonderen Befund.

1) Politzer: Labyrinthbefunde bei chron. Mittelohreiterungen. (Arch. f. Ohrenheilk. Bd. 65, S. 161.) 
Beiderseitige Radikaloperation an 28. Aug.:

Link e Seite: Periost narbig: Knochen sehr sklerotisch. Das Antrum, in etwa $2 \mathrm{~cm}$ Tiefe erreicht, enthält etwas schmieriges Fluidum und mißfarbige Granulationen; der umgebende Knochen mißfarben, angenagt. Von der Pauke ist zunächst nichts aufzudecken. Man kommt mit der abgebogenen Sonde von oben her $3 / 4 \mathrm{~cm}$. in die Tiefe und zwar unter das Niveau der hyperostotisch verdickten unteren Gehörgangswand. $\mathrm{Um}$ diese tiefe Bucht zugänglich zu machen, wird die untere Gehörgangswand schichtweise verdïnnt. Gehörknöchelchen in neugebildete Knochenmassen eingebettet: Vom Hammer fehlt ein Sttick des Manubriums, vom AmboB der lange Schenkel; ihre Oberfläche ist rauh, angenagt. Extraktion eines Sequesters (?) aus der Pauke. Allmählich bekommt die Wundhöhle normale Konfiguration. Am Bogengange ist nichts zu sehen; intra operationem sollen sich nystagmusartige Zuckungen gezeigt haben. Die häutige Gehörgangswand ist stenosiert, für eine Sonde gerade durchgängig. Plastik, Naht, Tamponade.

Rechte Seite: Retroanrikularschnitt mit Exzision der alten Narbe. Periost, soweit von solchem überhaupt zu reden - es handelt sich im wesentlichen um Narbenmassea, die mit dem Knochen verwachsen sind -, abgeschabt. In der Höhe der oberen Gehörgangswand, etwa $1 / 2 \mathrm{~cm}$ hinter dem Meatix, eine Fistel, in die man mit der Sonde ca $1 \mathrm{~cm}$ vordringen kann. Hintere Gehörgangswand hochgradig hyperostotisch. Extraktion eines den Aditus erfüllenden Sequesters. Abmeilelung von Hyperostosen am Paukendach und der Paukenvorderwand, bis endlich eine leidliche Mittelohrkonfiguration zustande kommt. Manubrium des Hammers fehlt. Plastik, Naht. 29. Aug.: Temperaturanstieg bis $37,5^{\circ}$. Sehmerzen in den Ohren; Verband rechts blutig durchtränlzt. Beim Verbandwechsel zeigt die Wunde gutes Aussehen; kein Belag, kein Fieber. Nachmittags ist Patient sehr unruhig. 30. dug.: Temperatur $38,8^{\circ}$. Einlauf und Ol. Ricini.

1. Septbr.: Vormittags Anstieg bis 40,6 ${ }^{\circ}$, abends $39^{\circ}$. Sensorium klar; kein Nystagmus, keine Nackenstarre. Klagt über Stechen in der Brust; in der rechten Axillargegend leichte Dämpfung.

2. Septbr: Temperatur steig't von $38^{\circ}$ anf $40^{\circ}$, fällt nachts wieder auf $38^{\circ}$. Kopfschmerzen; Patient macht einen etwas benommenen Eindruck. Atmung beschleunigt, Puls frequent.

3. Septbr. : Temperatur $39,6^{\circ}$; Patient ist benommen; keine Nackenstarre, kein Nystagmus. Pupillen gleich weit, reagieren. Verbandwechsel: Wunden zeigen mäbige, nicht übelriechende Sekretion. Lumbalpunktion: Liquor vermehrt und getrübt, enthält Streptokokken. Abends $40,6^{\circ}$. Patient ist sehr unruhig; Clysma von Chloralhydrat.

4. Septbr : Temperatur 41,5\%. Starke Nackenstare; Bewaßtsein geschwunden; Leib kahnförmig: eingezogen; Beine im Kniegelenk in Beugestellung.

Blutuntersuchung auf Bakterien negativ.

5. Septbr.: Exitus letalis.

Sektion am 6 . Septbr. 22 Stunden post mortem.

Auszug aus dem Sektionsprotokoll des pathologischen Instituts: Otitis media chron. duplex; Meningitrs purulenta; Dilatatio ventr. utr. cordis; Cyanosis hepatis et lienis; Tracheo-Bronchitis purulenta; Abscessus tonsill. sin; Vulnus reg. mastoid. et ossis petrosi utr. ex operatione.

Linke Lunge an der Spitze adhärent, ödematös, blutreich; in der Spitze verkalkte Taberkel; rechte Lunge in Unter- und Mittellappen adhärent, Spitze wie links.

Gehirn: Dura mit den Sehädeldach fest verwachsen. Sinus enthalten sämtlich flüssiges Blut; Pia zeigt nirgends den normalen Glanz, sondern Trülbungen verschiedener Stärke. Am Gyrus paracentralis zahlreiche pacchionische Granulationen und eiterige Beläge: Pia verdickt. Auf der Höhe beider Scheitellappen annähernd symmetrisch je ein 5 Pfennigstück großer; eiteriger Belag. An der Basis des linken Schläfenlappeus dicke eiterige Auflagernig. Eine ebensolche findet sich am Oberwarm des Kleinbirns sowie am Inf undibulum. An Pons und Basis sonst wur Trubung und feine eiterige Auflagerung in geringer Menge. A m

Archiv f. Ohrenheilkunde. 74. Bd. Festschrift. 
stärksten ist die Pia an der Basis des Kleinhirns verändert, indem sie hier eine derbe sehnige Membran vou ähnlicher Beschaffenheit wie die Dura darstellt.

Selstion der im Zusammenhange mit der Dura entfernten Schläfenbeine: Rechts eine kleinwallnußgroße Wundhöhle, die bei Fehlen der hinteren Gehörgangswand Mittelohräume und Gehörgang gemeinsam umfaßt. Trommelfell und äuBere Gehörknöehelchen fehlen; Stapes ist nicht zu sehen. Die Wundhöhle ist mit einer roten granulierenden Membran ausgekleidet und mit eiterigem Sekrete bedeckt. Knochenwandungen der Mittelohrräume intakt.

Mikroskopisch erscheint die Schleimhant der Pauke mäßig geschwollen, ziemlich stark kleinzellig infiltriert, mit Plattenepithel, an einzelnen Stellen mit Zylinderepithel bedeckt. Stapes, Paukenfenster unverändert. Häutiges Labyrinth gut erhalten; speziell Cortisches Organ, Otolithenmembran des Sacculus, Ampullarorgane gut ausgeprägt; Cupula terminalis der Ampullen erhalten.

Linkes $0 \mathrm{hr}$ : An der Rïckfiäche der Pyramide zeigt die Dura etwa in der Mitte zwischen Apertura externa meatus auditorii intemi und Sulcus sigmoideus ein erbsengroßes Loch mit unregelmäBig zackigen Rande, durch das man mit der Sonde in einen zwischen Dura und Pyramide gelegenen, mit eitrigen Bröckeln erfullten Hohlraum gelangt; der Grund der Hohle ist; soweit makroskopisch erkembar, mit einem Durablatte ausgekleidet. Übrige Dura und Knochen intakt. Mittelohrräume and Wundhöhle makroskopisch wie rechts.

Bei der mikroskopischen Untersuchung (Schnittführung horizontal mit etwas nach außen geneigter Schnittebene) erscheint die Mittelohrschieimhant stark infiltriert. Der Stapes ist aus seiner normalen Lage luxiert und zwar ist der vordere Rand der Platte in das Vestibulum hneingetrieben. Vom Ringband ist nichts mehr wahrzunehmen; statt dessen sieht man Granulationsgewebe aus der Pauke in den Vorhof eindringen Auf der Vestibularseite der Stapesplatte liegt massenhaft Eiter. Runde Fenstermembran völlig in Granulationsgewebe anfgegangen, das die Nische wie die scala tympani der Schnecke als einheitliches Polster erfüllt: Nur bei GiesonFärbung sind in dieser Granulationsmasse einzelne parallel verlaufende Bindegewebsfasern, offenbar die Resto der Fenstermembran, nachzuweisen. Schnceke: Im Vorhof sabschnitte reichliche Eitormassen, besonders in der Nachbarschaft des runden Fensters; dagegen nimmt die Eitermenge weiter nach der Spitze zu sehr schnell ab. Einzelheiten des Cortischen Organs sind nicht za erkennen; Membrana Corti, Stria vascularis relativ gut erhalten ReiBner'sche Membran liegt der tympanalen Wand des ductus cochloarís aut. Innerer Gehörgang von Eiter und jungèm Granulationsgewebe erfüllt; besonders seine hintere Wand entlang zieht bis in den Fundus hinein eine lange Exsudatstraße; endostale Auskleidung stark infiltriert. Im Fundus liegt ein großer Eiterklumpen; Eiterkörperchen finden sich des weiteren im canalis spiralis des Mediolus, von der Basis nach der Spitze zu an Menge abnehmend.

Die endolymphatischen Räume des Vestibulums mit Eiter erfülit; Nervenendigungen im sacealus und utriculus, sowie in den Ampullen zerstört, Otolithenmembram, cupula terminalis nícht zu differenzieren. Der ductus endolympathicus ist in seinem ganzen Verlaufe mit Eiter erfült, der von innen nach außen an Menge zunimmt; am stärksten ist die Exsudatmenge im Saccus endolymphaticus. Die knöcherne Wand des aquaeductus vestibuli zeigt abgesehen von auffallend zahlreichen Ausbuchtungen keine wesentlichen Veränderungen, um so stärkere dagegen die häutige Wand des ductus codolymphaticus. Im intratemporalen Telle ist das Epithel fast überall abgestoben; die bindegewebige Unterlage des Epithels ist infiltriert und blutreich, $z$. T. dureh Odem stark verbreitert, die bindegewebigen Maschen stellenwetse sehr vergröfert. Auch im Saceus ist die epithelỉale Auskleidung zum Teil zerstört, doch streckenweise noch gut erhalten. Das Lumen des Saceus, normaler Weise ein kapillarer Spalt, ist erweitert und euthält Eiter in Form größerer und kleinerer Klampen und Ballen. Die 
Wandungen des Saccus sind im ganzen verdickt; das vordere, den Knochen aufliegende Durablatt ist von diesem (wahrscheinlich artefiziell bei der Präparation) abgelöst. Während die inneren Bindegewebslagen beider Durablätter noch ziemlich straff gefẗgt erscheinen, sind die äußeren Lagen, offenbar durch Austritt von Lymphflüssigkeit, durch größere und kleinere $\mathrm{Z}$ wischenräume von einander getrennt, auseinander gerissen. Die Wandung ist yon zahlreichen. $z$. T. mit weilen Blutkörperehen gefülten Lymphspalten durchsetzt und weist stellenweise kleinzellige Infiltrationsherde auf. An einer Stelle erscheint das hintere Blatt eingerissen; an der Einrißstelle ist das Durablatt wie aufgefasert. - Der aquaeductus cochleae enthalt geringe Hitermengen.

Resumierend können wir das Tatsächliche des Falles in zwei Sätzen ausdrücken : Bei einem Manne mit doppelseitiger chronisch er Mittelohreiterung entwickelt sich im Anschlusse an die beiderseitige in einer Sitzung vorgenommene TotalaufmeiBelung eine in 8 Tagen zum Tode führende eiterige Leptomeningitis. Mikroskopisch findet sich auf der einen Seite eine Labyrintheiterung.

Bei der Epikrise handelt es sich zunächst um die auch für unsere speziellen Erörterungen wichtige Frage: Ist die Meningitis durch die Labyrintheiterung vermittelt worden oder auf anderem Wege, etwa durch Vermittlung eines Extraduralabszesses entstanden; wie hat sich der Übergang der Eiterung vom Mittelohr nach den Gehirnhäuten vollzogen? Noch bevor durch die mikroskopische Untersuchung die linkss eitige Labyrintheiterung aufgedeckt war, konnte ein direkter Durchbruch aus dem Mittelohre, etwa durch das Tegmen oder die Hinterwand nach der mittleren bzw. hinteren Schädelgrube ausgeschlossen werden: Der Knochen und die denselben bedeckende Dura war bis anf die Stelle am Saccus endo. lymphaticus intakt und unverändert. Damit fiel auch von vornherein die Möglichkeit fort, daB die Labyrintheiterung retrograd, also erst im Anschlusse an die anf direktem Wege entstandene Meningitis selcundär sich entwickelt hat. Wir haben also als erste sicher feststehende Tatsache anzunehmen, daß bei der Entstehung der tödlichen Meningitis die Labyrintheiterung die vermittelnde Rolle gespielt hat.

Nun galt es, in zweiter Reihe festzustellen: Wie ist die Beteiligung des Labyrinths zustande gekommen und zu welchem Zeitpunkte? D. h. handelte es sich um eine alte, im Anschlusse an die Operation exacerbierte und propagierte Labyrintheiterung oder hat sich diese erst in den 8 Tagen zwischen Operation und Exitus entwickelt? Um zunächst den zweiten Teil der Frage zu beantworten, so kann bei Berücksichtigung der im Labyrinthe vorgefundenen mikroskopischen Veränderungen wohl kaum ein Zweifel darüber obwalten, daß die Eiterung als eine ganz frische, 
akut entstandene aufzufassen ist. Abgesehen davon, dab klinisch keinerlei Symptome vorhanden waren, die auf eine Labyrintheiterung hätten bezogen werden können (vgl. Krankengeschichte), läßt das Mikroskop alle jene Veränderungen vermissen, die wir bei länger dauernder Labyrinthitis, oft schon nach wenigen Wochen, beobachten können, wie Bindegewebs- und Knochenneubildungen. Soweit die feineren Teile des häutigen Labyrinths nicht durch die Eiterung zerstört sind ${ }^{1}$ ), sieht man sie relativ gut erbalten, so die Stria vascularis, die Membrana Corti, stellenweise sogar die Pfeilerzellen; nirgends finden sich degenerative oder sonstige Veränderungen sekundärer Art, wie sie bei länger bestehender Labyrinthitis nicht auszubleiben pflegen. Wir müssen also entscheiden: Die Labyrintheiterung bat sich erst nach der 0 peration entwickelt.

Schwieriger schon erscheint die Beantwortung des ersten Teils der Frage, welche Momente wir für den Einbruch des Eiters ins Labyrinth verantwortlich zu machen haben. Hier kann uns vielleicht die Berïcksichtigung der Durchbruchsstelle die Antwort erleichtern. Der Einbruch ist - das ist nicht ohne Bedeutung - auffallender Weise durch beide Paukenfenster gleich. zeitig erfolgt. Ein mehrfacher Einbruch ist an sich nicht wunderbar und auch schon vielfach beobachtet worden, nämlich dann, wenn es sich um Einschmelzung der knöchernen Labyrinthkapsel an verschiedenen Stellen handelt, wenn, wie es in solchen Fällen meist geschieht, die Labyrinthentzïndung langsam fortschreitet und wenn, während sich die erste Einbruchsstelle durch Granulationsgewebe schließt, sich gleichzeitig anderwärts ein zweiter Durchbruch vorbereitet. Denkbar ist es ferner, daß eine schwere akute foudroyant verlaufende Infektion, wie Scharlach, die Labyrinthkapsel an mehreren Stellen gleichzeitig arrodiert. Schlieblich können mehrfache Fisteln auch dadureh zustande kommen, daß eine Labyrintheiterung wieder nach außen durehbricht. In unserem Falle jedoch liegt die Sache ganz anders. Es handelt sich um eine ganz frische Labyrintheiterung, akut entstanden nach einer Totalaufmeißelung; das runde Fenster ist durchbrochen, der Stapes luxiert. Man denkt sofort an ein Trauma. Zweifellos wurde dies hier durch die Operation gesetzt.

1) Daß es sich bei der Zerstörung dieser Teile nicht um PräparationsArtefakte oder postmortale Veränderungen handelt, lehrt ein Vergleich mit dem anderen, in derselben Weise behandelten Schläfenbeine, bei dem das häutige Labyrinth in seinen Details gut erhalten war. 
Es handelte sich, wie uns die Krankengeschichte lehrt, um einen hochgradig sklerotischen Knochen; die Wandungen des Mittelohrs waren außerdem durch mächtige Hyperostosenbildung ganz außergewöhnlich verdickt. Die Meißelerschïtterung war also eine sehk starke; dazu kam die erschwerte Orientierung, da durch die zahlreichen Hyperostosen die normale Konfiguration der Mittelohrräume verändert bezw. verdeckt war, schlieBlich die lange mehrstiundige Dauer der doppelseitigen Operation. Alle diese Momente mögen mitgewirkt haben, eine Verletzung an den Paukenfenstern zu erleichtern oder einen bereits drohenden Durehbruch zu vollenden. Ich sage "vollenden", denn wir können annehmen, daß der Durchbruch durch die Fenster bereits vorbereitet, die runde Fenstermembran bezw. das Ringband schon zur Zeit der Operation durch die Eiterung verdünnt oder z. T. durchbrochen bezw. dureh Granulationsgewebe ersetzt war, da sie in 8 Tagen kaum so vollständig einschmelzen können, wie wir es hier sahen. Wir müssen danach annehmen, dah die Labyrintheiterung anch ohne die Operation früher oder später zustande gekommen wäre. Hätte man nun durch sofortiges Eingreifen am Labyrinth die letale Meningitis verhüten können? Nach der jetzt vielfach geltenden Meinung, bei Labyrintheiterung möglichst aktiv vorzugehen, wäre in unserem Falle die Indikation zur Eröffnung des erkrankten Labyrinthes gegeben. Nun waren aber die Erscheinungen nach der Operation durchaus nicht so eindeutio, daß sie uns auf eine bestimmte Seite hätten führen können ${ }^{1}$ ); man hätte also beide Labyrinthe d. h. auch ein völlig gesundes eröffnen müssen. Vor allem aber zeigt uns ein Vergleich der Sektionsbefunde an den Meningen mit denen am Labyrinthe, dab die Überschwemmung der Arachnoidealräume mit infektiösem Materiale in einer Rapidität erfolgt ist, die jeden Eingriff aussichtslos gemacht hätte. Die Veränderungen an der Kleinhirnpia (s. oben) machen es zweifellos, daß sie nicht viel jüngeren Datums als die im Laby. rinthe sind, daß sie nicht erst in den letzten Tagen entstanden sind, sondem schon zu einer Zeit, in der man auf Grund der vorhandenen Symptome zum Eingriff berechtigt war, weit entwickelt waren, vielleicht schon unmittelbar nach der Operation eingesetzt hatten. Der Durchbruch des Eiters durch das Labyrinth muß sich mit unaufhaltsamer Schnelligkeit, der Transport

1) Jetzt müßte man in ähnlich liegenden Fällen versuchen, mit Hilfe der von Barany gegebene Direktiven die erkrankte Seite heranszufinden. 
der Erreger im Labyrinth- uud Hirnwasser so rapid vollzogen haben, daß Labyrinth- und Arachnoidal-Eiterung fast gleichzeitig einsetzten. Die Temperatursteigerung am Tage nach der Operation ist das erste Zeichen der einsetzenden Meningitis gewesen.

Schließlich haben wir uns in unseren epikritischen Auseinandersetzungen mit der fiir unser Thema wichtigsten Frage zu beschäftigen, an weleher Stelle der Übergang des Eiters vom Labyrinthe auf die Meningen erfolgt ist. Da die Labyrinthkapsel nach den Schädelgruben zu überall intakt, der Knochen nirgends arrodiert war, bei der kurz dauernden Labyrintheiterung auch kaum in nennenswertem Maße eingesehmolzen sein konnte, kommt nur einer der präformierten Wege in Betracht $d$. b. entweder die Nervenkanäle des Akustikus oder einer der beiden Aquaedukte oder endlich mehrerer dieser Wege gleichzeitig. Was sagt uns hierüber der anatomische Befund? Alle drei Passagen waren mit Eiter erfüllt; am meisten der meatus internus und der canalis spiralis des Modiolus. Darnach käme also der innere Gehörgang in erster Reihe als die Stelle des Durchbruchs in Betracht. Nun zeigt uns aber eine sorgfältige mikroskopische Untersuchung folgendes auffallendes Verhalten dieser Stelle: Am stärksten ist die Eiterung im Meatus selbst, dagegen nimmt sie im Modiolus, und zwar von der Basis nach der Spitze zu immer mehr an Mächtigkeit $a b$, und ist in der Schnecke selbst mit Ausnahme des Vorhofsabschnittes ziemlich geringfügig. Des weiteren sehen wir im Meatus das Exsudat z. T. schon in junges Granulationsgewebe umgewandelt, während in Modiolus nur kleinzellige Herde, also Granulationen jüngeren Datums, vorhanden sind. Wir köunen uns diese Differenzen nur dann erklären, wenn wir annehmen, daß der Eiter von den Meningen den Nerven entlang: erst in den Meatus internus und von da aus weiter nach dem Modiolus vorgedrungen ist, diese Passage also in umgekehrter Richtung durchschritten hat, als wir anfangs vermutet haben. In der Tat erinnerte mich das mikroskopische Bild sofort an mehrere Befunde, die ich bei der Genickstarre-Labyrinthitis habe konstatieren können. Nachdem also in unserem Falle hier die Meningen vom Labyrinthe her auf einem noch $\mathrm{zu}$ erörternden Wege mit infektiösem Materiale ïberschwemmt worden sind, hat die eiterige Meningitis ihrerseits wieder durch Vermittlung des Nervenkanals, wie so häufig, zu einer zweiten, gewissermaßen retrograden Infektion des Labyrinths gefübrt. 
Da der Meatus internus ausscheidet, kommen die Aquädukte in Betracht. Nun ist im allgemeinen der aquaeductus cochleae aus Gründen, die ich bereits oben angedeutet babe, ein direkterer und bequemerer Verbindungskanal zwischen dem Arachnoidealraum und den Binnenräumen des Labyrinths, als die Vorhofswasserleitung. Trotzdem werden wir in unserem Falle den letzteren als den Infektionsweg annehmen müssen und zwar aus folgenden Erwägungen: Wie in vielen anderen Fällen war auch in unserem Falle die Beteiligung der einzelnen Bezirke im Labyrinthe an der Eiterung eine verschiedene; die Haupteitermassen fanden sich im Vorhof, während sie von der Schnecke einigermaßen reichlich eigentlich nur den Vorhofsabsehnitt erfüllten. Ferner: Abgesehen von der Cisterna perilymphatica enthielten die perilymphatischen Räume viel weniger Eiter als die endolymphatischen, ein Verhalten, das wir bei einer Genickstarre-Labyrinthitis im Beginn öfters beobachten können. Vor allem aber - und das ist das Hauptmoment, das uns deutlich auf den aquaeductus vestibuli hinweist - fanden sich die makroskopisch wie mikroskopisch am meisten ins Auge fallenden Veränderungen am Saceus endolympbaticus und an den benachbarten Partieen der Kleinhirnoberfläche. Wir müssen nach dem Sektionsbefunde annehmen, daß es zunächst zu einer Eiteransammlung zwischen den Durablättern an der apertura externa des aquaeductus, also zu einem sogenannten Empyem des Saccus gekommen ist, daß dann infolge des entzündlichen Reizes das hintere Blatt des Duralsackes mit der Kleinhirnpia verklebt ist, daß es hier zur Infektion der Arachnoidealräume gekommen ist, und daß dann bei der Herausnabme des Gehirns 1) an dieser Stelle infolge der Verklebung der Kleinhirnpia mit der Dura ein Einriß in letztere erfolgt ist und ein Stück des äußeren Durablattes mit der Kleinhirnoberfläebe im Zusammenhang geblieben ist. Daher die Angabe im Protokoll, daß die Pia an der Basis der linken Kleinhirnhemisphäre „eine derbe sehnige Membran von äbnlicher Beschaffenheit wie die Dura darstellt", daher weiter das Loch im hinteren Blatte des sonst uneröffneten Saccus.

Wir können also, glaube ich, mit an Gewißheit grenzender Wahrscheinlichkeit annehmen, daß hier die Infektion der Meningen vom linken Labyrinthe aus auf dem Wege des ductusendolymphaticus dureh Vermittelung eines Saceus-Empyems erfolgt ist.

1) Ich selbst habe der Gehirnsektion nicht beigewohnt. 
Ich hielt es für notwendig, die mikroskopischen Details dieses Falles genau und ausführlich wiederzugeben, weil bisher diese und äbnliche für die Pathologie der Labyrintheiterungen so wichtigen Fälle dermaßen oberflächlich geschildert sind, daß sie kaum als absolut einwandsfrei gelten können, daß sie jedenfalls von der Kritik (Boesch, W agener) an verscbiedenen Punkten mit Erfolg angegriffen werden konnten. Auch der Fall Politzers 1), den Wa gener als den einzigen gelten läßt, bei dem einwandsfrei ein vom Labyrinthe ansgehendes Saccus-Epyem beohachtet ist, ist doch nicht so ausführlich geschildert, daß wir uns ein rechtes Bild von den vorliegenden Veränderungen machen könnten. Die Forderung Passow ${ }^{2}$ ), in allen solchen Fällen sich nur auf die mikroskopische Untersuchung zu verlassen, ist zweifellos vollkommen berechtigt; keinesfalls dïrfen wir anf Grund eines operativen oder makroskopisch-anatomisehen Befundes die Diagnose "Saccus-Empyem" stellen, wenn wir seben, wie schwierig es ist, selbst bei genauer histologischer Untersuchung über das prae und post, den ursächlichen Zusammenhang der einzelnen Verënderungen sich Rechenschaft abzulegen.

Wollen wir daher in derartigen Fällen die diagnostischen Scbwierigkeiten bei der mikroskopischen Untersuchung überwinden und die Veränderungen am ductus endolymphaticus in ihrer pathologischen Bedeutung richtig beurteilen, so müssen wir uns vor allem über die normalen anatomischen Verhältnisse klar sein: Es wird daher am Platze sein, hier einige topographische und morphologische Bemerkungen anzuknüpfen.

Der den ductus endolymphaticus enthaltende Knochenkanal, der aquaeductus vestibuli, geht von der fossula sulciformis, einer feinen länglichen schlitzförmigen Öffnung im hinteren unteren Teil der medialen Vestibularwand, vor und unter der gemeinschaftlichen Eintrittsstelle des oberen und hinteren vertikalen Bogengangs, aus and zieht in einem ganz leicht gekrïmmten, mit der Konvexität nach hinten und auBen gerichteten Bogen nach unten, lateral und hinten. Der Kanal ist ziemlich frontal gestellt, und zwar bildet die Ebene, in der er gelegen ist, mit einer durch die obere Pyramidenkante gelegten Vertikalebene einen Winkel von etwa

1) Politzer: 1. c. Fall 2.

2) Verhandl. der Deutsch. otolog. Gescllsch. Wien 1906. (Diskussionsbemerkung Passows zu dem Hins berg'schen Referate). 
30 Grad. Um ihm also im mikroskopischen Schnitte möglichst in toto und im Zusammenhange mit dem Saccus $\mathrm{zu}$ erhalten, wäre es am zweckmäßigsten, die Schnittriehtung ziemlich frontal, etwa unter 30 Grad die Pyramidenkante schneidend, zu wählen. Da aber diese fast frontale Schnittführung für die Übersicht über das übrige Labyrinth, speziell für den Zusammenhang von Meatus internus und Schnecke nicht sehr geeignet ist, wählt man in zweckmäBiger Weise Horizontalschnitte; hierbei erhält man den gröBten Teil des intratemporalen Stückes rom Aquädukt in einem Schnitte, wenn man die Ebene desselben gegen die Pyramidenkante lateralwärts etwas neigt, allerdings nicht im Zusammenhange mit dem Saceus. Durch Serienschnitte läßt sich aber dieser Zusammenhang leicht rekonstruieren.

Der Aquäduktusi) mündet bekanntlich an der Rückfläche der Pyramide in einer schlitzförmigen Öffnung von variabler Form und Größe. Gewöhnlich wird die Öffnung von einer flachen Knochenschale dachziegelartig gedeckt, von der nach unten vorn medial und unten hinten lateral je eine feine, manchmal kaum bemerkbare Knochenkante zieht. Zwischen diesen Knochenkanten, also unterhalb der Apertura externa des Aquäduktus, liegt eine bald mehr, bald minder ausgeprägte Delle, die sich nach dem suleus sigmoideus zu abflacht, nach vorn in eine zur fossa jugularis verlaufende Furche übergeht und in die sich zwischen die erwähnten Knochenkanten der Saccus sehmiegt.

Auch die Lage der Apertura externa im Verbältnis zu den benachbarten Gebilden ist noch nicht ganz konstant. Sie liegt zwischen Apertura meatus interni und sulcus sigmoideus und zwar je nach Breite und Tiefe des letzteren entweder in der Mitte zwischen beiden oder dem Sulcus etwas näher in einer Linie, die den oberen Rand der Apertura meatus interni streift und von da dicht unterhalb des hinteren Bogengangs durch den Sulcus gezogen wird.

Knöcherne Umrandung des Kanals: Die Labyrinthkapsel reicht bekanntlich an der Stelle der apertura externa aquaeductus vestibuli bis an die Oberfläche der Pyramide; der umgebende Knochen zeigt deshalh im ganzen Verlaufe des Kanälchens das Gefüge der Labyrinthkapsel, d. h. festen, marklosen Knochen mit knorpelhaltigen Interglobularräumen, die nach hinten zu an Menge abnehmen und in der Nachbarschaft der

1) Nach Politze r 5-6 mm lang, 0,25 mm breit. 
apertura externa ganz verschwinden. An manchen Präparaten sieht man jedoch in der Umgebung der Apertura spongiösen Knochen; namentlich reichen von unten und außen kleinere und größere Markräume, bisweilen sogar Zellen, bis in die unmittelbare Nähe der Öffnung heran, finden sich fast regelmäßig speziell unter dem Boden des Saccus, sodaß es immerhin möglich ist, daß die Angabe, es könne von da aus, also direkt vom Knochen, mit Umgehung des Aquäduktus, zu einer Eiterung im Saccus bezw. einer Pachymeningitis an dieser Stelle kommen, zutrifft (Körner). Jedenfalls ist bei Saccuseiterung auf die Beschaffenheit des benachbarten spongiösen Knochens zu achten.

Die Knochenwand des Aquäduktus ist in keinem Falle, den ich daraufhin untersucht habe, ganz glatt und gerade, sondern zeigt, namentlich im mittleren Teile des intratemporalen Abschnitts, bald mehr, bald weniger zahlreiche Ausbuchtungen, unregelmäBig zackige Grenzlinien, die bei oberflächlicher Betrachtung leicht als das Resultat eines Arrosionsprozesses gedeutet werden könnten. Es fehlten aber stets die mikroskopischen Anzeichen eines solchen. - Wie verhält sich nun normaler Weise der in dem knöchernen Kanal gelegene ductus endolymphaticus und seine intrakranielle Fortsetzung der Saccus endolymphaticus? Bekanntlich bildet der ductus endolymphatiens zwei Wurzeln, die vom Sacculus bezw. vom Utriculus ausgehen und sich noch vor ihrem Eintritt in die Labyrinthkapsel mit einander vereinigen und zwar in der Weise, daß der eigentliche ductus rom lateralen Teile des Sacculus ausgeht und bald nach seinem Austritte aus diesem den ductus utriculo-saceularis in spitzem Winkel aufnimmt. Das perilymphatische Gewebe begleitet dabei den ductus bis hart an die Labyrinthkapsel, ohne anscheinend in diese hineinzugehen. $O b$ es nicht doch mit der bindegewebigen Grundlage des ductus in anatomischem Zusammenhange steht, vermochte ich nicht zu entscheiden.

Die Wandung des intratemporalen Teiles des ductus besteht aus einem einschichtigen flachen Epithel und einer bindegewebigen Grundlage. Letztere setzt sich wieder aus zwei Schichten zusammen, einer mehr lockeren maschigen und einer äußeren straffer gefügten, periostähnlichen Lage. Die Oberfläche der DuctusAuskleidung ist nie ganz glatt, sondern zeigt regelmäßig an einzelnen Stellen größere und kleinere pilzartige oder knopfförmige Exkrescenzen, die in das Lumen hineinragen und häufig nur durch einen dünnen Stiel mit der Unterlage zusammenhängen. 
Sie haben dieselbe Struktur wie die übrige Wand, nur erscheint das Epithel etwas flacher, die einzelnen Zellen wie anseinandergezogen, und das bindegewebige Netz viel weitmaschiger. Häufig kommen die Oherflächen zweier solcher an gegenüberliegenden Stellen befindlicher Exkrescenzen mit einander in Berührung, bisweilen ziehen sich direkte Brücken d. i. mit Epithel überkleidete Stränge von einer Wand nach der gegenüberliegenden; so daß das Lumen dadurch wesentlich verengt erscheint. Überhaupt zeigt das Lumen conform mit der wechselnden Dieke seiner Wandung keine konstante Weite und bildet bald einen kapillaren Spalt, bald seheint es bei zahlreicher Strangbildung mehrfach geteilt, bald ist es so weit, daß es den Durchschnitt eines membranösen Bogengangs um das mehrfache übertrifft; eine solche Verbreiterung des intratemporalen Teiles findet sich außer an der Apertura auch noch ziemlich regelmäßig am Scheitelpunkte des Konvexbogens, den er bildet.

Das Lumen ist auch an normalen Objekten nicht immer leer; sehr häufig finden sich in ihm abgestoßene und gequollene, vakuolisierte oder sonstwie veränderte Epithelzellen, z. T. wohl als postmortale Erscheinung. Sind diese Epithelzellen, wie es häufig geschieht, zu einem Klumpen zusammengeballt, dann glaubt man beim ersten Blick, es mit Eiter zu tun zu haben. Man hat also bei Verdacht einer Aquaedukt-Eiterung immer an dies „normale" Vorkommen abgestoßener Epithelzellen zu denken.

Wesentlich anders ist die histologische Beschaffenheit der Wand des intrakraniellen Teiles d. b. des Saccus endolymphaticus. Das Epithel zwar bleibt dasselbe, es setzt sich kontinuierlich vom eigentlichen Aquaedukt auf die innere Oberfläche des Saceus fort und verleibt derselben ein glattes glänzendes Aussehen. Die eigentliche Wand dagegen wird von der Dura gebildet und zeigt deshalb die Beschaffenheit der letzteren d. h. straffe parallelfaserige Bindegewebszüge, die unter dem Epithel besonders dicht gefügt sind. Die beiden Durablätter, die den Sack bilden, sind insofern etwas von einander verschieden, als das vordere, dem Knochen anliegende Blatt etwas schmäler ist, dafür aber aus straffer gefügten Bindegewebsfasern besteht als das hintere.

Die Breite bezw. Länge des Saccus beträgt nach Politzer im Minimum 5 bezw. 8 , im Maximum 9 bezw. $15 \mathrm{~mm}$. Für gewöhnlich bildet sein Lumen einen kapillaren Spalt; die ihn ihm enthaltene Flüssigkeitsmenge ist also ziemlich gering. Auch wenn 
ich die Injektionsflüssigkeit mit beträchtlichem Drucke in den Sack trieb, ließ sich derselbe nie weiter als bis zur Größe einer kleinen Bohne auftreiben. Da er nun - von der Öffnung nach dem Aquaedukt abgesehen - als ein allseitig geschlossener Sack anzusehen ist, erscheint es unwahrscheinlich, dab er, wie vielfach behauptet wird, zum Ausgleich von Druckdifferenzen im Labyrinthe dienen soll; sein Volumen und infolgedessen sein Inhalt dürfte wohl immer konstant sein. Außerdem wäre seine Rolle als Druckregulator sehr überflïssig, da ja der aquaeductus cochleae diese Funktion in viel bequemerer Weise verrichten kann. Physiologische Bedeutung kommt dem aquaeductus vestibuli nicht $\mathrm{zu}$; wir haben ihn als ein rudimentäres Gebilde aufzufassen, denn die Entwicklungsgeschichte nnd die vergleichende Anatomie lehren uns, daß er aus einer Umwandlung des bei der ersten Abschnürung des Labyrinthbläschens sich bildenden Stieles hervorgeht. Bei bestimmten Tierspezies z. B. bei Eidechsen, wo er sich in zahlreichen langen Ausläufern bis unter die Hant verliert, mag ihm vielleicht die oben erwähnte Funktion zukommen, beim Menschen jedenfalls kann er diese nicht mehr besitzen.

Aus dem gleichen Grunde, nämlich auf Grund der Tatsache, daß er allseitig abgeschlossen und nur wenig ausdehnungsfähig: ist - seine Wandung besitzt ebensowenig elastische Fasern in nennenswerter Menge wie die Dura an anderen Stellen - glaube ich auch nicht, daß er bei Eiteransammlung in seinem Innern bis zu Kirschgröße, aufs vielfache ausgedehnt werden kann, wie vielfach behauptet wird. 1) Es ist mir kein Zweifel, dab hier Verwechslungen mit großen Extraduralabszessen an der hinteren Pyramidenfläche vorliegen. Nun wird von verschiedener Seite angenommen, dab der Saccus keineswegs einen allseitig abgeschlossenen Hohlraum darstellt. So hat Rüdinger ${ }^{2}$ "verschieden lange akzessorische Kanälchen gefunden, welche an verschiedenen Stellen von der Wand des Fundus ausgehen und dann in mehrere Schenkel geteilt nach den subduralen Lymphspalten der Dura sich fortsetzen." Diese Kanälchen sind nach thm rudi. mentäre Homologa jener Gebilde, die, wie oben erwähnt, bei

1) Die Angabe Schulzes (Arch. f. Ohrenheilk. Bd. ว̃, S 70), daß es sich in seinem Falle (kirschgroBer, voll mit Eiter erfüllter Sack, der an 2 Stellen eingerissen ist) um ein Saccus-Empyem handelt, wird von Wag en er bezäglich ihrer Beweiskraft angezweifelt.

2) Sitzung der Gesellsch. f. Morphologie und Physiologie in München; 6. Dez. 1887. 
verschiedenen Tieren in der mannigfachsten Größe und Form vorkommen, und als Abflußwege der Endolymphe aufzufassen. Die Annahme, daß der Saccus blind endigt, wäre also nach $R \ddot{u}$ dinger nicht haltbar. In neueren Arbeiten habe ich diese Angaben $R$ üdinger nirgends bestätigt gefunden, und auch ein so vortrefflicher Beobachter wie Politzer, dem diese Kanäle doch wohl kaum entgangen wären, zitiert diese Befunde $\mathrm{R} \ddot{\mathrm{u}}$ dingers nur, ohne sie durch eigene zu bestätigen. Ich habe diese Kanäle in meinen Präparaten nicht auffinden können. Wohl fanden sich in der Wandung des Saccus zahlreiche Lymphspalten, die besonders in dem Falle von Empyem durch ihre starke Füllung mit weißen Blutzellen deutlich hervortraten, aber niemals Kanäle, die als Ausläufer des Saccus aufzufassen waren. Nur folgender Befund erinnerte mich an die Angaben $R$ üdingers: Bisweilen gingen von der vorderen Saccus-Wand und zwar immer in unmittelbarer Nachbarschaft der Apertura des Aquaeductus zwei bis drei Dura-Fortsätze aus, die sich in den Knochen der Pyramide einsenkten und eine kurze Strecke parallel zum Aqaedukt nach vorn verliefen. Es handelte sich aber immer um solide Durastränge, die niemals ein auch noch so kleines mit dem Saccus kommunizierendes Lumen aufwiesen. Zuckerkandl ${ }^{1}$ ) bat bei seinen Injektionsversuchen ebenfalls kleine Kanälchen in der Dura sichtbar machen können: $\mathrm{Er}$ sagt darüber folgendes: "Ich darf nicht unterlassen beizufügen, daß in gut gelungenen Fällen die Injektionsflüssigkeit stets auch in drei bis vier, selbst über $2 \mathrm{~mm}$ breite, aber lange Kanäle überging, die vom Fundus der Wasserleitung ausgingen und gleichfalls zwischen den Schichten der Dura mater verliefen; eine Kommunikation mit Blutgefäßen oder mit den Arachnoidealräumen gehen sie nach dem, was ich gesehen, ebensowenig ein, als der A quaeductus im Ganzen." Er gibt selbst an, daß es sich hierbei vielleicht um Artefakte gehandelt hat. Auf Grund meiner Untersuchungen möchte ich daran festhalten, daf eine physiologisch oder pathologisch bedeutsame Kommunikation $\mathrm{zwi}$ schen Ductus endolymphaticus und Arachnoidealräumen nicht existiert. Schon dies Moment schränkt die Häufigkeit eines Übergangs der 'Eiterung vom Labyrinth nach den Gehirnhäuten auf dem Wege der Vorhofswasserleitung gegen-

1) Zu ckerkandl: Über die Vorhofswasserleitung des Menschen. (Monatsschr. f. Ohrenheilk. Bd.10, S. 81). 
über demjenigen dureh die Nervenkanäle und den Aquaeductus cochleae gewaltig ein. Dazn kommt noch ein weiterer Umstand, der jenen Übergang ersehweren muß. Bei zahlreichen Fällen von Labyrintheiterung, und zwar sowohl vom Mittelohr, wie von den Meningen aus induzierten, bei Fällen, die ich histologisch untersucht habe, und bei denen ich dem Aquaeductus vestibuli meine Aufmerksamkeit widmete, sah ich, daß es trotz Anfüllung des Labyrinths mit Eiter niemals zu einem SaccusEmpyem (obigen Fall ausgenommen) oder auch zu nennenswerter Eiteransammlung im Aquaedukt selbst kam. ${ }^{1}$ ) Dies lag daran, daß der enge Kanal sich sehr schnell durch neugebildetes Bindegewebe, ja manchmal durch Knochenapposition schlob und so dem weiteren Vordringen des Eiters hirnwärts eine Schranke setzte, Bindegewebigen Versehluß der Vorhofswasserleitung findet man vielfach bei Taubstummen als Resultat einer abgelaufenen Labyrinthentzündung. Im allgemeinen dürften also die Meningen bei Labyrintheiterung vom Aquaeductus vestibuli ans nicht bedroht sein, nur bei sehr foudroyanten Eiterungen, wie in dem geschilderten Falle, ist der Durchbruch an dieser Stelle möglich.

Wir werden annehmen können, daß es sich in vielen der als Aquaedukt-Eiterung bezw. Saccus-Empyem beschriebenen Fälle um ganz andere Prozesse gehandelt hat; wir werden mit Wagener behaupten, dab, wenn auch nicht alle, so doch sebr viele einer Kritik nicht standhalten. Haben Fälle, die blob kliniseh, operativ oder makroskopisch beobachtet sind, bezüglich der erörterten Fragen überhaupt keinen Wert, so wird man aber auch bei der Deutung mikroskopischer Befunde recht vorsichtig sein und sich der verschiedenen, von mir oben auseinandergesetzten Täuschungsmöglichkeiten erinnern müssen.

1) Einzelheiten werden von mil demnächst bei anderer Gelegenheit beschrieben werden. 